\title{
Response to "Ten Misconceptions that Dentists have about Treating Obstructive Sleep Apnea"
}

Roy V. Hakala, DDS, FAGD

Diplomate of the American Board of Craniofacial Pain Diplomate of the American Board of Dental Sleep Medicine Diplomate of the Academy of Clinical Sleep Disorders Disciplines

"Ten Misconceptions That Dentists Have About Treating Obstructive Sleep Apnea" by Dr. Gail Demko in the July issue of the JDSM raises several concerns:

Dr. Demko discusses the increased mortality and comorbidities associated with sleep-disordered breathing (SDB) but then recommends against dentists screening all patients, even though adding relevant screening questions to a medical history costs essentially nothing. The American Dental Association and other professional groups, including the Minnesota Board of Dentistry, have recommended that dentists screen every dental patient for SDB. Dr. Demko's concern that screening patients for OSA may lead to overtreatment is curious. Hopefully, she does not think this way about screening for other disorders such as periodontal disease or hypertension.

Dr. Demko is critical of the use of cone beam computed tomography (CBCT) in the diagnosis and treatment of patients with SDB. Although a cone beam scan alone cannot determine the presence or absence of SDB, it can reveal conditions such as nasal septal/turbinate/sinus obstructions, tonsillar and adenoidal hypertrophy, faulty tongue posture, temporomandibular joint misalignment and condylar degeneration, and many other concerns. These conditions may require referral before treatment, can reveal the likelihood of occlusal changes with oral appliance treatment, determine that a patient is simply unsuitable for oral appliance treatment, and provide invaluable assistance in diagnostic and treatment decisions. In addition, CBCT exposes the patient to much less radiation than the conventional panoramic or fullmouth series of radiographs that Dr. Demko recommends.

Dr. Demko denies a relationship between orthodontic treatment and the risk of the development of obstructive sleep apnea (OSA). However, a comprehensive conference paper concluded that constricting an upper dental arch clearly increases OSA development, and that "orthodontic expansion of the maxillary dentition is often effective as a fundamental treatment of OSA." 1

Finally, Dr. Demko denies a relationship between OSA and gastrointestinal reflux disease (GERD). Although both are complicated conditions with multiple etiologies and each can occur independently of the other, the literature has established a strong connection between the two. In fact, the prevalence of GERD has been shown to be at $58 \%$ to $62 \%$ in patients with OSA., ${ }^{2,3}$

Dr. Demko is entitled to her opinions, and her paper is clearly an opinion piece consistent with its sensationalist format. Publishing and promoting it as "must read" scientific literature misinforms Academy members and does a disservice to their patients. It also provides insurers with additional ammunition to use in denying necessary treatment.

\section{CITATION}

Hakala RV. Response to "Ten Misconceptions that Dentists have about Treating Obstructive Sleep Apnea". J Dent Sleep Med. 2019;6(1)

\section{REFERENCES}

1. Maeda K, Tsuiki S, Inoue Y. (2010). Does dental arch size affect obstructive sleep apnea? https://www.researchgate.net/publication/266771657_Does_Dent al_Arch_Size_Affect_Obstructive_Sleep_Apnea. Accessed December 7, 2018.

2. Green BT, Broughton WA, O'Connor JB. Marked improvement in nocturnal gastroesophageal reflux in a large cohort of patients with obstructive sleep apnea treated with continuous positive airway pressure. Arch Intern Med. 2003;163(1):41-45

3. Herr J. Chronic cough, sleep apnea, and gastroesophageal reflux disease. Chest. 2001;120(3):1036-1037. 


\section{SUBMISSION AND CORRESPONDENCE INFORMATION}

Submitted in final revised form September 17, 2018 Accepted for publication September 27, 2018

Address correspondence to: 2550 University Ave West, Suite 143N, St. Paul, MN 55114; Email:

drhakala@mncranio.com

\section{DISCLOSURE STATEMENT}

The author has no financial interest or any other conflicts of interest involving any products, materials, procedures, or intellectual property described in this letter, or in the Journal of Dental Sleep Medicine article to which his letter responds. 